\title{
Aproximación sociológica \\ a las necesidades y al consumo \\ de los mayores
}

\section{Ester Bódalo Lozano \\ Universidad de Murcia \\ ebodalo@um.es}

\section{RESUMEN}

En este artículo hemos analizado las distintas concepciones de necesidades en el segmento de población de más de 65 años, pudiendo afirmar que si bien este grupo de población tiene necesidades comunes al resto de los grupos de edad, también es posible que necesite otra serie de bienes y servicios que le diferencien de otros segmentos de población.

Tratamos de resaltar cómo el factor tiempo influye en los hábitos de consumo de las personas mayores, ya que éstas han vivido momentos o situaciones diferentes derivados de la edad, siendo el resultado final del total de los momentos vividos y de la situación personal en que vive a cada momento.

Por último, se trata de ver cómo los efectos de la generación a la que pertenecen, así como los diferentes ciclos de vida familiar, influyen en su posición hacia el consumo, haciendo énfasis en que cada vez se trata de un segmento más numeroso de consumidores mayores.

Palabras clave: Ancianos, Consumo, Necesidades Sociales, Bienestar Social. 


\section{INTRODUCCIÓN}

Una reflexión sobre las necesidades y el consumo de los mayores es, en definitiva, un debate entre lo público y lo privado. Muchos de los problemas del mayor no se resuelven sólo en la sociedad de consumo, sino que muchas de las necesidades provienen de su situación objetiva (económica, familiar), y otras de las propias limitaciones de los servicios públicos para atenderlas, a lo que se le añade que es un número creciente de personas. La empresa privada que busca su nicho de mercado, cada vez se da más cuenta de que estas necesidades no se atienden por el Estado, por lo que surgen empresas privadas que tratan de satisfacer esta parte de la demanda que el Estado no atiende.

El envejecimiento demográfico constituye hoy en día uno de los fenómenos sociales con más relevancia en nuestro país. En los últimos años han proliferado los estudios que tienen por objeto llegar a un conocimiento de las causas, las dimensiones y las repercusiones de este fenómeno social.

Pero el estudio de este fenómeno no debe agotarse en los aspectos demográficos; éstos constituyen un elemento introductorio para ocuparnos después de quiénes son los auténticos protagonistas: las personas mayores. Debemos intentar llegar más allá en el conocimiento de todos los temas o aspectos que influyen en sus actitudes, comportamientos y estilos de vida. En lo que a este artículo se refiere, los que tienen que ver con sus actitudes hacia lo económico, sus comportamientos ante el consumo, sus hábitos de compra y su socialización en la cultura del consumo.

Cabe destacar que existe una escasez de estudios que aborden, no sólo desde el punto de vista nacional, sino también regional, uno de los temas que más repercusiones sociales va a tener en nuestro futuro, como son las influencias de este colectivo de mayores sobre la economía y el consumo. Como señalan algunos especialistas (Navarro, 1987: 501), «la consideración de factores sociales en la literatura del marketing es escasa (...) Así la interacción con otros individuos, los grupos de referencia, la clase social o la familia se consideran en sus efectos atomizados, en tanto se refleja en ese esquema de respuestas individuales». Desde una perspectiva más global y sistémica (la sociológica), se trata de conocer las implicaciones de la sociedad de consumo y las construcciones sociales sobre las personas mayores.

Si embargo, señalamos cómo en otros países ya ha habido estudios interesados por el segmento de consumidores mayores. En el caso de España es un tema que está despertando recientemente un interés creciente; sin embargo, en Estados Unidos hay un número importante de investigaciones y artículos sobre el asunto. Por hacer referencia a algunos, 
destacamos «Selling to Senior» (Noel, 2001), donde se discute por qué muchas empresas no comercializan sus productos a los consumidores más viejos y se plantea cómo la economía de Estados Unidos, esforzándose, puede invertir esta tendencia. Otro estudio interesante es el llevado a cabo por Mike Duff (1998), «Seniors», donde se centra en el poder del consumidor mayor americano. Richard C. Levantal (1997), en su trabajo «Aging consumers and their effects on the market place», examina los valores que son cruciales para tener en cuenta en un mercado envejecido. Los trabajos del profesor Ildefonso Grande Esteban, de la Universidad Pública de Navarra, han sido pioneros en España.

Otro exponente de su mayor importancia es el referente al ámbito de la publicidad, donde se detecta un mayor número de productos de consumo específicos para las personas mayores donde éstas muestran actitudes más vitalistas o la creciente presencia de mayores en los anuncios publicitarios (Sánchez Vera y Bódalo, 2000). Por lo tanto, los especialistas en investigación de mercados y un buen número de industriales están viendo en los mayores un segmento merecedor de ser tratado conforme a su ascendente cuota de participación en el negocio, en el cual durante muchos años la publicidad y el consumo habían sido un campo donde se trataba de exaltar la belleza, la juventud, la alegría, el optimismo, y donde la figura del mayor aparecía de manera secundaria, sin ser los auténticos protagonistas del mensaje (se utilizaban para dar consejos, para resaltar el contraste entre lo moderno y lo tradicional, para aportar experiencia o sabiduría, etc.). El consumidor mayor forma parte de la sociedad, pero en un mercado crecientemente segmentado se impone el considerar al mayor con creciente fuerza y en donde habrá de ser tomado más en consideración en las próximas décadas.

Es manifiesto que en la sociología española escasean los trabajos que aborden al mayor desde la perspectiva del consumidor y que pongan a éste en relación con el sistema social. Como antecedente más destacado en España para estudiar el fenómeno cabe citar, entre otros, el libro de SECOT (Seniors para la Cooperación Técnica) de 1995 con el título Las actividades económicas de las personas mayores. También han sido de interés los congresos de empresas y entidades que se dirigen al sector de las personas mayores. Por último, cabe señalar el reciente estudio llevado a cabo por el Instituto Nacional de Consumo, dirigido por el profesor Manuel Martín Serrano, de la Universidad Complutense de Madrid: La tercera edad y el consumo (Instituto Nacional de Consumo, 2000), donde a través de una encuesta de ámbito nacional se trata de conocer al segmento creciente de consumidores de más edad.

En sociedades como la nuestra, que están inmersas en un proceso de envejecimiento colectivo, el cambio individual se transforma en nuevas necesidades que se convierten en retos y oportunidades para las Administraciones públicas y para las empresas. Hay 
que prepararse frente a este nuevo reto demográfico, porque raramente se piensa en los mayores desde la perspectiva del consumo para ellos. El perfil de los mayores como consumidores y usuarios está cambiando muy rápido. Su influencia y potencial social, político y económico no va a parar de crecer en muchos años, a pesar de que siga siendo realidad la presencia de muchos mayores que se adecuan a los estereotipos dominantes (Moragas, 1991).

\section{LAS NECESIDADES Y LOS MAYORES DESDE LA PERSPECTIVA TEÓRICA}

Hablar de las necesidades y deseos de los mayores supone plantearse el concepto del bienestar, ya que el componente fundamental de éste se halla en la satisfacción de las necesidades. Habrá que plantearse, pues, la cuestión de si los mayores como colectivo tienen unas necesidades específicas. Todas las teorías de las necesidades aceptan al menos el carácter instrumental de las mismas y el hecho de que algunas sean universales; quiere decir que trascienden los gustos personales y las culturas particulares. Las diferentes teorías se pueden clasificar dependiendo de la interpretación del término «necesidad».

- Teoría de las necesidades objetivas, porque «su especificación teórica y empírica es independiente de las preferencias individuales»(Doyal y Gough, 1991: 49). Por lo tanto, todas las necesidades de los mayores no serán diferentes a las de otro grupo de edad.

- Teoría de las necesidades subjetivas; supone la expresión subjetiva de las necesidades por parte de cada individuo particular. Por ello los mayores no tendrán necesidades como colectivo, sino como personas individuales.

- Teorías universalistas; éstas consideran que las necesidades en sí son iguales para todo el mundo. Ello implica que no hay diferencia en función de raza, sexo, edad, grupo social, cultura, etc., del individuo o grupo que se esté tratando.

- Teorías relativistas; éstas afirman lo contrario, es decir, que las necesidades vienen definidas en función de la cultura, el sexo, la edad, la raza, etc., del objeto de estudio que estemos considerando. En este sentido, los mayores tendrán necesidades relativas a su propia edad.

Si hacemos una combinación de los cuatro elementos anteriores, quedarían reflejados de la siguiente manera en este cuadro (Aguiar, Pérez Yruela y Serrano, 1995). 


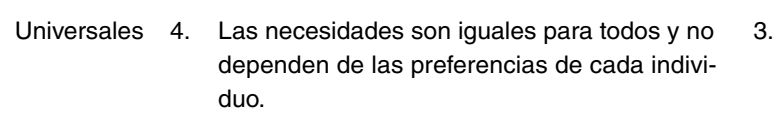

Universales 4. Las necesidades son iguales para todos y no 3.

Relativas 1. Las necesidades no dependen de las preferencias individuales pero están determinadas por la sociedad.
2. Las necesidades dependen de las preferencias de los individuos y están socialmente determinadas.

De esta manera, partiendo de este cuadro podemos situar las distintas teorías de las necesidades en alguna de las casillas.

1) Teorías objetivas-relativistas. Donde las necesidades se pueden especificar de manera teórica y empírica de forma objetiva, es decir, sin tener en cuenta las preferencias subjetivas de cada uno, pese a ser éstas relativas. Esto supone, según la teoría de la que se trate, que las necesidades dependerán: bien de una situación histórica concreta (marxismo), bien de una cultura, de la comparación entre distintos grupos sociales, etc. Aunque las necesidades sean objetivas (definibles, medibles), no son iguales para todos; son diferentes para cualquier cultura, época o grupo social.

2) Teorías subjetivas-relativistas. Sólo los propios individuos saben definir y expresar sus necesidades, las cuales son relativas a cada sociedad, edad, sexo, cultura concreta. Entre estas teorías cabe destacar:

a) Economía del bienestar ortodoxa. En ella las necesidades objetivas se sustituyen por los términos preferencias y demandas. Si partimos de la supuesta soberanía del consumidor, el cual toma las decisiones en un mercado del que está totalmente informado, las necesidades serían las preferencias de un conjunto de personas que generan una demanda que ha de ser satisfecha. Está claro que tales preferencias están determinadas socialmente (Doménech, 1985).

b) Las necesidades son específicas de los grupos sociales. Esta teoría cobra fuerza en los años sesenta con movimientos sociales tales como el feminismo, el antirracismo, el anticolonialismo, etc. Sólo los individuos que pertenezcan a una raza, pueblo, grupo o sexo concretos saben cuáles son sus necesidades. En este caso, el subjetivismo individual de la economía ortodoxa se sustituye por un subjetivismo colectivo.

c) Argumentos fenomenológicos/posestructurales: las necesidades se construyen social o discursivamente. Para Laclau y Mouffe (1987): «Los intereses (...) son un producto 
social y no existen con independencia de las conciencias de los agentes que los portan". Así, las necesidades no tienen una existencia objetiva fuera de las mentes individuales; es a través de los discursos sociales como se revela su existencia. La fenomenología considera que las necesidades, la realidad social toda, en definitiva, es un constructo social dinámico que depende de la práctica de grupos específicos profesionales (Smith, 1980).

3) Teorías subjetivas-universalistas. Por la propia definición que hemos aportado de los diferentes tipos de necesidades, se trata de un tipo de teorías imposibles ya que las necesidades en sí no pueden ser, a su vez, subjetivas y universales.

Siempre existirá una polémica entre necesidades relativas y subjetivas u objetivas y universales. Por lo tanto, hay que dejar claro si nos referimos al espacio de los bienes, de sus características, de las necesidades (funcionamiento/capacidades en el lenguaje de Sen, 1984) o de las utilidades.

Visto así, si de lo que se trata es de estudiar las necesidades de las personas mayores, podemos decir que serán relativas y subjetivas en el espacio de los bienes y de las utilidades, pero serán objetivas y universales (absolutas) en el espacio de las necesidades.

Las teorías objetivas se enfrentan a las relativistas, haciendo equiparable el concepto de necesidad a una serie de condiciones universales. En este sentido, la obra de los autores Len Doyal e lan Gough (1991) pone en relación el término necesidades básicas y el grave daño o perjuicio que supondría no satisfacerlas. Para quitar elementos de subjetividad, estos autores afirman que una persona sufre un daño cuando se ve impedida de participar plenamente en la sociedad a la que pertenece. Así, la satisfacción de las necesidades básicas es una precondición necesaria que posibilita la acción. La salud y la supervivencia física, así como la autonomía, serían las necesidades básicas objetivas y universales que posibilitan el desarrollo mínimo del individuo en una sociedad.

Pero para lograr la satisfacción de las necesidades básicas es preciso contar con una serie de características universales de los bienes, servicios, actividades y relaciones. A ese conjunto de propiedades universales, con las que hemos de contar, Doyal y Gouhg las denominan necesidades intermedias. Éstas serían las siguientes: alimento y agua adecuados, vivienda adecuada, ambiente de trabajo que no entrañe peligro, medio ambiente que no entrañe peligro, cuidados sanitarios adecuados, infancia segura, relaciones primarias significativas, seguridad física, seguridad económica, control de la natalidad y embarazo, seguros y educación básica. 
Los bienes específicos que satisfacen las necesidades básicas e intermedias son particulares de cada cultura, pero las necesidades como tales son objetivas y universales. A partir de estas necesidades (básicas e intermedias), estos autores desarrollan un complejo conjunto de indicadores sociales transculturales, los cuales permitirían analizar el grado de satisfacción de las necesidades de un país o región.

Sin embargo, tampoco hay que olvidar la situación objetiva de los mayores, donde en el caso de España presenta importantes deficiencias económicas (con respecto a otros grupos de población); se trata de un colectivo donde un escaso número de mujeres ha estado incorporado al mercado laboral, al que hay que prestar más atención en cuanto a las necesidades asistenciales y a los problemas de soledad que sufre, y a las crecientes demandas de salud. Por tanto, los mayores como grupo social, aunque participan también de las necesidades universales, tienen también necesidades específicas en mayor medida que otros, tales como de salud, prestaciones económicas y atención.

\section{LOS SISTEMAS DE NECESIDADES. LA EMERGENCIA DEL CONSUMIDOR MAYOR}

Vistas todas las posiciones anteriores, si nos apoyamos en una teoría objetiva y universal, en ningún momento se podrá hablar de necesidades específicas de la tercera edad, o de cualquier grupo social. Si, por un lado, consideramos que el bienestar de una persona está en función del grado de satisfacción de sus necesidades, el bienestar de las personas mayores no se verá diferenciado del de ninguna otra persona. Es decir, en el ámbito de las necesidades (básicas e intermedias) se puede hablar de necesidades objetivas y universales que los mayores comparten con cualquier persona. Se puede decir, sin embargo, que los miembros de la tercera edad necesitan más o menos bienes de cierto tipo, o incluso diferentes y específicos de cualquier grupo de edad.

Según la anterior reflexión, no parece conveniente hacer de la edad una variable absoluta; la clase social, el sexo o la raza son variables que también hay que tener en cuenta para satisfacer las necesidades que todo grupo social, o de edad, comparten. Esto nos puede llevar a la conclusión de que este tipo de teorías universales y objetivas necesitan mucha cantidad de información de la situación exacta en la que se encuentran las personas para llegar a una satisfacción óptima de sus necesidades. Así, a modo de ejemplo, no se pueden equiparar las necesidades de un anciano de clase alta que haya previsto su jubilación con las de un anciano del medio rural que viva de lo que le ofrece la tierra. Es un error frecuente considerar a la tercera edad como un grupo homogéneo y considerar sus necesidades como tales, tal y como han puesto de relieve distintos especialistas (Sánchez Vera, 
1993a: 48). Como señala Gil Calvo (1992), es necesario segmentar a la tercera edad en distintos niveles para obtener la mayor información posible de su situación y distribuir los recursos eficazmente y de manera justa. Dicha segmentación debería hacerse atendiendo, entre otros criterios, a:

a) La edad no es lo mismo una persona de 65 años que una de 80.

b) El género hay que distinguir entre hombres y mujeres de la tercera edad.

c) Los recursos es preciso conocer la procedencia de clase o estatus de la persona mayor.

d) El territorio y el hábitat, teniendo en cuenta que no es la misma situación la de los ancianos rurales que la de los urbanos, o de una ciudad de veinte mil habitantes que de dos millones.

e) Según el grado de acceso a redes interpersonales de solidaridad, como la situación familiar, o el acceso a redes de apoyo, etc.

De lo que se trata, en definitiva, es de adecuar la satisfacción de las necesidades de las personas mayores a la diversidad de sus demandas; para ello es preciso no considerarlas como un grupo homogéneo. Aunque sí es cierto que los mayores, pese a tener una serie de necesidades específicas propias de su condición (disminución de los ingresos y de la salud, pérdida de roles, etc.), también comparten junto al resto de la sociedad una serie de necesidades que hemos calificado como universalistas y objetivas o absolutas y atemporales, es decir, que todos los seres humanos tenemos necesidades comunes básicas, y que su especificación teórica y empírica es independiente de las preferencias individuales. En este sentido es clásica la teoría de Maslow (1954), que, desde el punto de vista de las necesidades absolutas o prioritarias y desde una parcela más psicológica, establece y describe una serie de necesidades que van desde las necesidades alimenticias y sexuales (prioritarias) a las de seguridad, posesión y amor, estima y autorrealización. Cada una de esas necesidades encontrará en las personas mayores aspectos comunes o compartidos, entre ellas y el resto de la sociedad, y a su vez específicos en su gradación.

En el aspecto de las necesidades, como hemos expresado con anterioridad, la cultura y el propio sistema económico y social juegan un papel muy importante, pues las múltiples maneras de satisfacer una necesidad en una sociedad concreta se convierten en una necesidad en sí misma y, lo que es más importante, la concreción de las necesidades no es posible hacerla si no es en referencia al sujeto y tomando en consideración su propia subjetividad. En cualquier caso, parece evidente que hay unos condicionantes objetivos de las necesidades (sistema, cultura, grupo), pero que éstas también tienen sesgos subjetivos e individuales.

El envejecimiento es un fenómeno en el que se entreveran una serie de procesos biológicos, psicológicos, culturales, económicos y sociales; por lo tanto, los cambios que experi- 
mentan los seres humanos son tanto de tipo biológico como de salud, de personalidad, de estilo de vida, de poder adquisitivo o de estatus social. Pero este conjunto de cambios no son experimentados de igual manera por todos los sujetos que comparten la misma edad, por lo que el proceso de envejecimiento se convierte en algo heterogéneo para el conjunto social. En esto radica la dificultad para la comprensión del envejecimiento.

El hecho de envejecer es algo inherente al ser humano; sin embargo, como fenómeno social, ha adquirido en las últimas décadas una serie de características que han hecho que sea más visible. Fundamentalmente, el aumento de la esperanza de vida y la disminución de la fecundidad han contribuido a que cada vez sea mayor el número de ancianos y que las probabilidades de llegar a la vejez sean cada vez más elevadas, dejando de ser éste un estadio de la vida reservado tan sólo a unos pocos.

El envejecimiento, hasta hace poco tiempo, era considerado como un proceso de deterioro físico inevitable e irreversible, donde el sujeto iba experimentando una serie de cambios físicos, motores o sensoriales. Pero estos cambios no eran universales, sino que cada uno de los sujetos los experimentaba de manera diferente, con igual, mayor o menor intensidad. El fenómeno de la vejez, como complejo que es, se ve afectado por una serie de factores que han de tenerse en cuenta para comprender la vejez en su realidad social. Por lo tanto, este fenómeno consiste, pues, en un proceso de adaptación individual o generacional a estos cambios, pero éstos no se producen de igual manera en los sujetos; aquí radica la dificultad de comprensión del fenómeno y que no pueda ser éste considerado como algo universal. Como hemos señalado, los acontecimientos históricos compartidos por cada generación, las experiencias individuales vividas por cada sujeto, o la pertenencia a una diferente cultura, hacen que existan diferentes «tipos de vejez».

\section{LAS NECESIDADES DE LOS MAYORES Y EL CONSUMO}

Cualquier análisis sobre el consumo remite al tema de las necesidades; éstas son siempre un dato que no se cuestiona, como algo natural dado eternamente, sin intentar comprender el qué, el cómo y el porqué de la necesidad, y su relación con el medio social, no como un falso problema de prelación del individuo o la sociedad, sino en la manifestación social de la necesidad.

Las necesidades se clasifican en dos tipos: las fisiológicas, enraizadas en la naturaleza humana, y las psíquicas, o secundarias, que tienen una génesis histórica en el sistema de producción industrial, el cual ha sabido descubrir y satisfacer esas necesidades, o crearlas artificialmente, y que hace que se relacione cada producto con una 
necesidad, negando los problemas que genera esa operación y, por consiguiente, su explicación.

Se puede hacer una aproximación al problema de las necesidades de consumo a condición de abandonar el planteamiento que pretenda definir las necesidades como reflejo de la naturaleza humana, cuya esencia, una vez descubierta, se convierte en el fundamento de un orden productivo y de un orden social. Las necesidades que son objeto del proceso de consumo, esto es, que tienen un contenido económico, «no son definidas por la naturaleza humana en general, sino por la solvencia, es decir por el nivel de ingresos del que disponen los individuos y por la naturaleza de los productos disponibles, que son, en un momento dado, el resultado de las capacidades técnicas de producción» (Althusser, 1969). De esta forma, las necesidades de consumo son definidas económicamente en el sentido de que dependen del grado de desarrollo técnico del sistema productivo, en cuanto a la cantidad y naturaleza de los objetos que puede producir, y de las relaciones sociales de producción, en la medida que determinan la distribución de los ingresos entre las clases sociales. Las necesidades económicas deben ser analizadas en relación con el sistema productivo que se monta para satisfacerlas.

En este sentido, la teoría marxista arroja una cierta luz. Marx, aunque no niega el componente biológico de las necesidades, las presenta condicionadas socialmente y, por lo tanto, con una historia. «Las necesidades naturales —el alimento, el vestido, la calefacción, la vivienda, etc. - varían con arreglo a las condiciones del clima y a las demás condiciones naturales de cada país. Además, el volumen de las llamadas necesidades naturales, así como el modo de satisfacerlas, son de suyo un producto histórico que depende, por tanto, en gran parte, del nivel de cultura de un país» (Marx, 1968). En esta línea, Paul Baran (1957), a propósito de una crítica del concepto de soberanía del consumidor, expresa que las necesidades de la gente son complejos fenómenos históricos que reflejan la interacción dialéctica de sus requerimientos fisiológicos, por una parte, y el orden económico y social prevaleciente, por otra.

La sociología, aunque no ha tratado sistemáticamente el tema, ha intuido un condicionamiento social de las necesidades o, indirectamente, del consumidor. Para Halbwachs (1970), «si nuestras necesidades son obra de la sociedad, sus principios (del mismo modo que su forma y sus objetos) no pueden explicarse más que por ella». Al igual que Marx, explica cómo la satisfacción de las necesidades orgánicas está revestida de formas sociales - gustos - que las vacían de su contenido primitivo hasta casi desvanecerse. «A través de un trabajo lento y metódico de descomposición, de limitación, y sobre todo de sustitución, la sociedad, vacía poco a poco nuestras necesidades de todo contenido orgánico primitivo, destruye la escala de los valores de las diversas satisfacciones fisiológicas, que tienden a 
establecerse espontáneamente en el individuo, tanto más cuanto menos integrado esté en la sociedad. La vida social enriquece la vida orgánica, abriéndole perspectivas nuevas e indefinidas; la complica y la refina».

Jean Baudrillard es, posiblemente, el que más lejos ha llevado el tema del análisis de las necesidades. Según él, hay que partir del hecho de que una teoría de las necesidades carece de sentido, ya que sólo puede haber una teoría del concepto ideológico de necesidad, y ello porque solamente desvelando las ideologías sobre el concepto de necesidad se puede llegar a una definición de las mismas, definición que tiene que basarse «ya no en absoluto, según la tesis naturalista-idealista, como fuerza innata, infusa, apetencia espontánea, virtualidad antropológica, sino como función inducida en los individuos por la lógica interna del sistema, más exactamente, no como fuerza consumidora liberada por la sociedad de la abundancia, sino como fuerza productiva requerida por el funcionamiento del propio sistema, su proceso de reproducción y de supervivencia. Dicho de otra manera: sólo hay necesidades porque el sistema las necesita» (Baudrillard, 1976).

La necesidad es un producto social, pero no solamente en el sentido de que constituya una costumbre o un hábito, sino porque todo elemento cultural revela una estructura social, es decir, un conjunto de relaciones sociales. Cuando una persona satisface una necesidad está realizando una acción social. Cada sociedad tiene una estructura de necesidades que viene definida socialmente, así como los mecanismos de su difusión o inculcación. Ambos forman una unidad que, a su vez, configura y define a la sociedad. En el proceso de satisfacción de necesidades, que al mismo tiempo es su creación, toda sociedad expresa mediante relaciones simbólicas su estructura social, y ese lenguaje simbólico que tiene por objeto reforzar la estructura social constituye la configuración externa de esa sociedad. En este sentido, la satisfacción de las necesidades de los mayores, con sus consiguientes actos de consumo, son una forma de relacionarse con el sistema social.

\section{TIEMPO Y CONSUMO, CONSTRUCCIÓN DE LOS HÁBITOS DE CONSUMO EN LOS MAYORES}

El consumo constituye una de las formas que tiene el individuo de relacionarse con el sistema social, y así les ocurre a las personas mayores. El consumo de éstas se verá condicionado principalmente por una serie de variables objetivas y subjetivas que nunca habrá que perder de vista. Entre las variables objetivas están: su nivel de ingresos, el estado de salud, y su situación familiar y patrimonial, su propia edad, el género. Entre las subjetivas están los valores y actitudes personales. En la medida en que todas estas variables sean más favorables y positivas, los mayores estarán más abiertos a las relaciones sociales y al 
trabajo, su abundante tiempo libre lo utilizarán para pasear, charlar con amigos, hacer la compra, la comida, etc. Por ello interactuarán en mayor medida con el sistema social; en una relación de feedback, recibirán más información del medio al tiempo que estarán más insertos en la sociedad de consumo, con actitudes más vitales y positivas, a la vez que serán tomados más en consideración por industriales, comerciales y expertos de mercado.

Entre las actividades que más realizan los mayores (CIS-IMSERSO, 1998) es cierto que destaca, por encima de todas, la audiencia de los medios de comunicación de masas: televisión $(96,9 \%)$ y radio $(71,4 \%)$, pero la proporción de los que realizan otras cosas que requieren una mayor actividad y, en definitiva, una cierta manera de relacionarse con la sociedad también es considerable. El $70 \%$ suele dar paseos, una proporción similar hacer la compra o recados, la mitad lee, uno de cada tres suele acudir a bares o a cafeterías, y uno de cada cuatro a clubes de mayores o establecimientos similares.

La salud es uno de los factores que más afectará al estilo de vida de los mayores y a la forma de relacionarse con su entorno; determinadas deficiencias les obligarán a permanecer en casa y, quizá, a gastarse el dinero en medicamentos o en personas que los cuiden. Según la Encuesta sobre Discapacidades, Deficiencias y Estado de Salud del INE (2001), un 32,21\% de los mayores de 65 años sufre alguna discapacidad, ocasionada en su mayor parte por algún tipo de enfermedad. De entre las enfermedades que más afectan a los mayores, según la Encuesta Nacional de Salud (1997), están la hipertensión arterial, que afecta a más de un $50 \%$ de las mujeres mayores y a casi el $40 \%$ de los hombres, y las enfermedades del corazón, que afectan en mayor cuantía a los varones (más de un $30 \%$ )que a las mujeres $(21,1 \%)$.

Para una mejor comprensión de la construcción de los hábitos de consumo de los mayores deberíamos tener en cuenta, entre otros, los siguientes aspectos:

- Efecto generación.

- Biografía personal (movilidad social).

- Situación económica y patrimonial.

- Situación y ciclo familiar (de su hogar y el de sus allegados).

- Percepción subjetiva de la situación económica personal y general de la sociedad.

- Actitudes individuales subjetivas ante el consumo.

- Grado de decisión personal con el que se relaciona respecto al consumo.

- Grado de autonomía física y mental o psicológica.

- Teoría del género.

En relación al concepto de generación son importantes las aportaciones de la obra de Mannheim sobre este aspecto, cómo un mismo acontecimiento es percibido de manera di- 
ferente en función de la edad del sujeto. La coexistencia de los acontecimientos tiene lugar en el tiempo cronológico, pero el único tiempo verdadero es el vivencial, el que cada individuo comparte sólo con sus coetáneos; cada generación vive «en un tiempo interior que en lo cualitativo es plenamente diferente al de los otros»; cada generación vive una época distinta y propia (Mannheim, 1993: 200). La generación tiene dos dimensiones: la de «verticalidad», en tanto que sucesión de descencientes, y la de «horizontalidad», como la coexistencia en un mismo momento de generaciones diferentes. De esta manera, un mismo producto referido al consumo va a tener un efecto distinto en la generación de personas mayores y en otra más joven (Sánchez Vera, 2001).

Desde el punto de vista metodológico, para conocer la construcción de los hábitos de consumo en el consumidor mayor sería deseable tomar en consideración que un sujeto ha pasado a lo largo de su vida por distintos momentos de la sociedad de consumo, y que cada uno de ellos los percibe a una edad, existiendo una continuidad en la trabazón de experiencias acumuladas (Sánchez Vera, 2001: 97). El esquema a seguir sería el siguiente:

A. Cronología de los fenómenos de consumo vividos por un sujeto a lo largo de su vida.

B. Edad personal a la que ha experimentado los distintos fenómenos de consumo.

Fases de consumo vividas:

Momento I (edad A1)

Momento II (edad B2) (A1+B2)

Momento III (edad C3) $(\mathrm{A} 1+\mathrm{B} 2+\mathrm{C} 3)$

Según este esquema, cada sujeto vive cada momento de consumo a una edad determinada, siendo producto del total de momentos vividos y de la situación personal en que vive cada momento.

Si una categoría merece ser enfatizada respecto al consumo, ésta es el tiempo, pues no sólo se trata del tiempo histórico, sino de las secuelas y de los efectos de éste en las distintas generaciones, en la sociedad y en los individuos. El tiempo (tempus), en tanto que temporada, está ligado a la caducidad, en el sentido amplio de los productos en la sociedad de consumo; así como también a la estacionalidad de los consumos en distintas estaciones (climáticas) del año, o en función de distintos periodos nacidos o recreados en la sociedad de consumo (Navidad, rebajas, promociones, efemérides diversas, etc.). Que la sociedad de consumo fagocita e instrumentaliza los procesos sociales y culturales en términos de dicho consumo es un asunto en el que ya nos han puesto en antecedentes y des- 
de distintas perspectivas los grandes especialistas (v.g.: Veblen, Bell, Friedman, Marx, From, Bourdieu, Baudrillard, etc.).

El paso del tiempo (y los sistemas de necesidades para cada momento social y personal) deja sus efectos sobre las distintas generaciones y sobre los individuos. En la sociedad, las "generaciones» tienen dos dimensiones: la «verticalidad», en tanto que sucesión de descendientes, y la «horizontalidad», como coexistencia en un mismo momento de generaciones diferentes. Así, sabemos que los hombres nacidos en distintos momentos viven y experimentan acontecimientos diferentes, y un mismo hecho o acontecimiento tiene efectos diferentes en función de la edad a la que se perciba, ya que existe un bagaje de experiencias acumuladas de las que somos producto. De esta forma, y referido al consumo, un mismo producto o una generación de ellos, como por ejemplo la telefonía móvil, va a tener un efecto distinto en una generación de personas mayores que en otra mucho más joven. No obstante, ello no quiere decir que el consumidor mayor vaya a quedar al margen de esos fenómenos. Con independencia de que las teorías del cambio social inciden en la distinta posición de los sujetos en razón de la edad ante un mismo fenómeno, sin embargo, es muy frecuente que, junto a la edad (generación), existan también otras categorías que a veces pueden cobrar la misma entidad que la propia edad. No es menos cierto que la existencia de algunos rasgos comunes entre los mayores, tales como la situación económica, que se deriva de ser en su mayoría pensionistas, está condicionando su posición respecto al consumo.

Pero, en cualquier caso, nos faltan estudios comparativos con suficiente entidad analítica para poder deslindar y clarificar con nitidez qué factores de los que afectan a las actitudes, a las percepciones y al comportamiento de consumo vienen determinados propiamente por la edad, y cuáles vienen determinados por otros factores (situación económica y patrimonial, familiar, laboral, educativa, género, etc.).

Las aportaciones de Pierre Bourdieu sobre los hábitos de consumo y sobre la sociología del gusto son extraordinariamente enriquecedoras, pues ponen sobre el tapete la incidencia del origen social y de la movilidad social individual sobre los hábitos de consumo (Bourdieu, 1988).

A nivel general, y con independencia de los procesos de movilidad social (si es que esto fuera posible), referido al consumidor mayor, podemos hacer las siguientes apreciaciones:

- La situación económica de los mayores tiende a degradarse cuando se llega a la condición de jubilado y/o de viudez. 
- Los mayores tienen cierta experiencia en adecuarse a una nueva situación económica, ya que a lo largo de la vida han pasado por distintas fases.

- Los mayores pueden introducir en mayor medida que otros colectivos cierta racionalidad y escepticismo al consumo, sin por ello dejar de ser un segmento creciente y rentable.

A través de las distintas fases de la vida, el sujeto ha ido cambiando sus hábitos de consumo, adaptando éstos a su situación económica y familiar y a la evolución de sus gustos y preferencias (cuando se está en disposición de poder elegir). El consumo es, fundamentalmente, un fenómeno familiar, y es difícil conocer con detalle la estructura del gasto en las distintas fases del ciclo de la vida de un sujeto. Diferentes estudios han puesto de relieve cómo cada una de las etapas o ciclos de vida familiar del sujeto lleva emparejada una serie de gastos (Ruiz de Maya, 1999). Con todo, existe una cierta orfandad de trabajos de investigación sobre el consumo familiar de los mayores en España.

Dado que muchos de los modelos teóricos sobre comportamiento de los consumidores utilizados por el marketing miran más los factores que impulsan a consumir un determinado producto y/o una marca concreta, es por esto que algunos autores entiendan que ese tipo de literatura (referido al marketing) deba ser denominada como de «comportamiento de compra» y no como de «comportamiento del consumidor» (Navarro, 1987: 500-501), pues los aspectos sociales del consumo son básicos y nunca deben ser reducidos a la parte psicológica. Las implicaciones y el estatuto teórico del consumo suponen toda una relación social tanto en la función como en la forma o «signo» del objeto de consumo (Pérez-Agote, 1978). De otra parte, desde el punto de vista metodológico, la demanda de objetos de consumo está entreverada con modelos sociales generales (Ortí, 1976), que no le son ajenos al consumidor mayor. Con respecto al consumo de las personas mayores en España, éste no podrá ser aislado de las diferentes características que tienen modelos de consumo más globales y su evolución específica (Alonso y Conde, 1994; Conde, 1994).

\section{TEORÍAS DE LA VEJEZY LOS HÁBITOS DE CONSUMO}

La situación social de la vejez se ve marcada por la confluencia de una serie de circunstancia que van a definirla. Entre las teorías sociales de la vejez, será «la teoría de la actividad» la que nos lleve mejor a comprender el tema de los mayores y del consumo, la cual se encuentra en el polo opuesto de «la teoría de la desvinculación». Havighurt y Albrech (1953) fueron los primeros que postularon que para tener un envejecimiento exitoso era necesario llevar una vida activa, lo cual supone reemplazar roles y actividades perdidos por otros nuevos. Para esta teoría, un alto grado de actividad social en los últimos años de la vida 
contribuye a una vida más feliz. Para estos teóricos, hay que evitar que el envejecimiento sea traumático; para ello hay que adoptar cualquier actitud que sea beneficiosa para el equilibrio del anciano. Las bases para un envejecimiento óptimo son: la actividad, la sociabilidad y la participación. La teoría de la actividad, al igual que las teorías de la competencia social y de la subcultura, se relacionan con el interaccionismo simbólico (Blumer, 1982). Por lo tanto, una de las bases fundamentales de todas ellas es considerar que los seres humanos desarrollan sus valores, significados y modos de actuar a partir de un proceso de comunicación social.

Otras teorías sociológicas, como la de la desvinculación, han puesto de relieve cómo hay un proceso de «desenganche» de los mayores del medio social (Cumming y Henry, 1961). A esa situación contribuye la pérdida de roles que supone el abandono del trabajo y la pérdida de poder adquisitivo (Gubrium, 1973), siendo ciertas y centrales las limitaciones estructurales que suponen los factores sistémicos en la construcción social de la vejez. Sin embargo, y aun existiendo importantes bolsas de pobreza entre los mayores (Alfageme, 1999), la mejora en su situación económica, tanto desde una perspectiva cronológica como con respecto a otros grupos sociales (Sánchez Vera, 2000), y las nuevas actitudes de éstos a vivir la vejez de manera más activa y menos estigmatizada, han contribuido a mejorar sensiblemente la inserción social de los mayores en España.

Quizá lo más característico del caso español haya sido la tardía pero decidida irrupción de los mayores en el mundo del consumo. La relación sujeto-sistema que se establece a través del consumo merece una lectura de la sociología que integre las perspectivas de dos ámbitos disciplinares raramente puestos en conexión: la sociología de la vejez y la sociología del consumo.

La vinculación al consumo es un «signo» (Baudrillard) y un deseo de inserción social. En este sentido, el consumidor mayor merece ser tenido más en consideración, con una perspectiva más holística —esto es, sociológica - que la que tiene la economía, que, desde su práxico devenir, se ha dado cuenta de manera decidida (sobre todo los especialistas en investigación de mercados) de la importancia de este segmento de consumidores. Con independencia de las limitaciones y frustraciones que puede generar y genera la sociedad de consumo, sin embargo, en la medida que los mayores manifiesten actitudes más vitalistas y positivas hacia la cultura económica y el consumo, podremos hablar de una mayor inserción social y de una calidad de vida más autónoma e independiente.

Al analizar los hábitos de consumo de los mayores en España, hemos de partir del principio de que cada año están entrando generaciones, y que cada vez en más cantidad han sido partícipes de la sociedad de consumo española. A modo de ejemplo, un sujeto que el 
año 2000 haya cumplido los 65 años, tenía 30 a mitad de la década de los sesenta, pero como la sociedad de consumo en España entró algo más tarde que en la mayoría de países de nuestro entorno socioeconómico, tal como señalan distintos especialistas en la materia (v.g.: Alonso y Conde, 1994; Conde, 1994: 147-148), los mayores actuales —con grandes diferencias según cohortes, género, tipo de hábitat y niveles económicos y de instrucción- ya conocieron algo tarde el fenómeno del consumo de masas. Por ello, en general, no son un colectivo imbuido plenamente de la mentalidad consumista, y de ahí que un cierto corte generacional deje a estos grupos etáneos alejados del consumo masivo e indiscriminado. Sin embargo, las generaciones nacidas a partir de mediados de los años cincuenta ya han vivido y se han socializado desde sus primeros años de vida con la sociedad de consumo.

\section{FASES DEL CONSUMO DE MAYORES}

Referido a las etapas en las que existen personas mayores dentro del hogar, las que siguen son las fases del ciclo y los bienes y servicios de consumo más usuales en cada una de ellas: 1) En la fase de Nido lleno 3 (matrimonios maduros con hijos dependientes), los bienes más usualmente consumidos eran los siguientes: educación universitaria, segundo automóvil, mobiliario y hogar, libros y revistas, viajes. 2) En la fase de Nido vacío (matrimonios mayores sin hijos viviendo en casa): mejoras del hogar, viajes, planes de pensiones, productos de inversión, servicios médicos. 3) Sobreviviente (personas mayores que se han quedo solas): transporte, productos farmacéuticos, ocio y recreación, viajes, servicios domésticos.

A partir de los datos existentes en España (INE), la clasificación por grupos poblacionales más parecida a la establecida por el comportamiento del consumidor nos dice que los hogares formados por personas de más edad, que suelen coincidir con las fases de «nido vacío II» (matrimonio sin hijos que convivan con ellos), «nido vacío IIl» (matrimonio con uno de los cónyuges jubilado) y supérstite solitario, gastan más que la media, en términos relativos, en los grupos G1 (alimentación, bebidas y tabaco), G2 (vestido y calzado), G3 (vivienda, calefacción y alumbrado) y G5 (servicios médicos y gastos sanitarios). Como ponen de relieve algunos especialistas, «sería deseable una mayor profundización en el consumo de los hogares en los que viven mayores, aunque esta tarea no siempre es fácil, pues en la ECPF del INE, los datos son por hogares y no por sujetos» (Grande, 1993: 31-32).

Lo que parece evidente, en cualquier caso, es el hecho de que el mayor tiene que aquilatar su gasto al extremo, pues ha de administrar con rigor su magra pensión. De otra parte, dispone de tiempo sobrado para dotar de la máxima racionalidad económica a su conducta de 
consumo. Por último, y a pesar de lo exiguo de las pensiones españolas y del pesimismo económico que determina el comportamiento del mayor, la toma de referencia con periodos anteriores de su propia situación económica en tanto que pensionista (no olvidemos la favorable evolución de las pensiones en España en los últimos diez años), y aún más la toma de referencia con respecto a generaciones predecesoras en la jubilación que pasaron todas las penurias, es un hecho relevante.

Sin embargo, y de manera paradójica, el mayor tiene que asumir y asume «lo económico» como uno de los contenidos y de los significados más afianzados en su cotidianeidad. De una parte, los temas de conversación frecuentes entre los mayores están referidos al dinero y a la administración de su economía doméstica, aunque están expropiados de ciertos conocimientos instrumentales imprescindibles. Este bajo nivel de instrucción de los mayores los hace frecuentemente desconfiados hacia las mudanzas que la vida económica moderna impone.

También hay que observar que la mejora en la «cultura económica» de los españoles ha afectado a los mayores. La cultura del ahorro es uno de los ejes centrales de cultura económica del anciano. Ya lo hemos dicho, el mayor, a pesar de su parca pensión, tiende a gastar lo imprescindible, y a veces menos; el resto lo ahorra o lo reparte entre sus familiares más allegados, con los cuales puede convivir o no. Cabe señalar el papel de la pensión de los abuelos en el mantenimiento de muchos hogares españoles. Éste es un factor en el que hay que insistir, sin que por ello tengamos que restar vitalidad al papel asistencial de la familia en España. En palabras de un experto, «los dineros de los abuelos, en general, son dineros bien administrados por una generación sobria y parca, que ahorra en sus libretas, para luego financiar juguetes a los nietos pequeños, entradas en la compra del piso para los nietos, y que tapan los agujeros de fin de mes de sus hijos (...) Es una generación que consume poco, y productos españoles, apagan la luz al salir, guardan las bolsas de plástico y las cuerdas y cintas de la tarta de cumpleaños. La gran función de las pensiones de la Seguridad Social es la redistribución económica y social. (...) los abuelos y abuelas españolas, con su consumo ordenado, son una demanda que genera desarrollo y, sobre todo, estabilidad económica. Son además un pacificador social, una memoria oral histórica (en el seno de la familia) que nos recuerda permanentemente de donde venimos (un país pobre)» (Gaviria, 1996: 98).

Pero, desde el punto de vista económico, hay un factor que en modo alguno debe pasar desapercibido: el papel de las pensiones en las economías domésticas. Como ponen de relieve diferentes investigadores (Pérez, 1998; Bazo, 1996), las pensiones de los mayores en España tienen una importante función económica complementaria para las familias. En algo más de un tercio de los hogares españoles entra una pensión de la Seguridad Social 
(casi ocho billones de pesetas al año, que desde los bancos y, sobre todo, desde las cajas circulan animando la economía española).

Un buen número de depósitos de ahorro a plazo proveniente de mayores se ha convertido en fondos de inversión. La disponibilidad de tiempo y el intercambio de opiniones entre los mayores en uno de sus temas preferidos, sus disponibilidades monetarias, hacen previsible un crecimiento de esta cultura económica. Pero, en cualquier caso y referido al ahorro, todo hace indicar que no es un cliente emprendedor, y mucho menos aventurero. Referido a este ahorro de los mayores, señala Moragas (1991:196-197) lo siguiente: «El Estado reconoce la importancia de las decisiones presentes de los futuros jubilados y trata de fomentar el ahorro con lo que aumenta el capital disponible para inversión, detrae dinero del consumo y controla la inflación (...), aunque (...) el propio ahorro se ve afectado por la inflación que devalúa en pocos años las pesetas ahorradas (...) debido a ello, la población puede preferir el consumo presente al ahorro y futura renta empobrecida, olvidando la previsión del futuro en aras del bienestar actual».

Ciertos elementos relacionados con la cultura del ahorro, como son las actitudes del mayor hacia la «reutilización» (sobre todo), la «recuperación» y el «reciclaje» de ciertos productos de su vida cotidiana, son de alguna forma elementos centrales de una cultura económica contraria al consumismo y al despilfarro, que a su vez forman parte del paradigma ecológico. Un reciente estudio realizado por la Comunidad de Madrid ha puesto de relieve una manifiesta colaboración de las personas mayores en las tareas de discriminación de papeles y vidrios. Con independencia de las anteriores conductas típicamente cívicas y ecológicas, también el mayor se familiariza con ciertas actividades de reparación y bricolaje, muchas de las cuales están ligadas a la estrechez económica con que tiene, debe o, simplemente, desea conducirse, y que le lleva, por ejemplo, a no llamar a operarios para pequeñas reparaciones domésticas. La disposición de tiempo libre le permite, igualmente, realizar de grado estas tareas domésticas y pequeñas reparaciones. Estas conductas son tanto masculinas como femeninas, aunque suelen ser más del primer tipo. La mujer, cuando queda viuda, también tiene necesidad de realizar frecuentemente actividades domésticas a las que no estaba habituada, y puede mostrar un comportamiento más dependiente que el varón en la realización de las mismas.

\section{LOS MAYORES: UN MERCADO EMERGENTE}

Una pregunta que debemos hacernos es si la categoría edad —referida al segmento de mayores de 65 años - representa por sí misma una categoría suficiente y con entidad propia, digna de ser tomada en consideración por los especialistas en comportamiento del consumi- 
dor. La respuesta a la cuestión anterior no es sencilla, a pesar de que exista un creciente interés por conocer las necesidades, las demandas y los anhelos de este consumidor.

Desde la perspectiva de los especialistas en introducir productos especializados que satisfagan las necesidades y demandas del consumidor mayor, así como desde la perspectiva de los especialistas en marketing e investigación de mercados, la respuesta no tiene duda: el segmento de consumidores mayores es numéricamente creciente y económicamente rentable. Nadie duda que la rentabilidad económica proviene a su vez, entre otros, de los siguientes factores:

- El potencial cuantitativo del segmento.

- La mejora experimentada en la situación económica de los mayores.

- La presencia de actitudes vitalistas que favorecen la integración social.

- La mejora en el nivel educativo.

- Las mejores condiciones de salud.

Sin embargo, los problemas más habituales con que tienen que vérselas estos profesionales — referido al consumidor mayor- están relacionados con la búsqueda de una adecuación entre fines (quién y qué) y métodos (mensajes y canales). En un mercado cada vez más segmentado y con mayores niveles de complejidad, el problema se plantea cuando la categoría «edad» se entrevera con otras categorías.

Ciertamente que la sociedad de consumo se presenta cada vez más fraccionada por subsegmentos especializados de consumidores (sociedad de públicos, en términos del profesor Castillo), y que atienden al juego de distintas categorías sociales y personales. Referido a la categoría edad, sin embargo, la oferta de los productos que atienden las necesidades de los mayores, así como la de aquellos otros productos que satisfacen necesidades y demandas sociales del conjunto de la sociedad pero que pueden ensanchar su mercado entre sujetos mayores, deben ser extremadamente escrupulosas a la hora de dirigirse al consumidor mayor y entreverar la edad con otras categorías sociales. En suma, se trata de vincular desde la lógica y desde la dinámica interna de la sociedad de consumo al sujeto mayor, cuestión ésta que a la postre es compleja y que desborda las pretensiones aproximativas de este trabajo. En cualquier caso, nadie duda que, desde la lógica interna del sistema, el consumo es un elemento de integración social de un colectivo «sistémicamente descolocado». A la postre, cuatro al menos son los problemas básicos a sortear por el mercado frente al consumidor mayor:

1) Que el consumidor mayor desea sentirse integrado en otros segmentos distintos al de la edad (género, clase y estatus, grupos de referencia, etc.). 
2) Que la categoría edad adquiere carácter regresivo (transposición de imágenes negativas sobre la vejez) y que, justamente en el caso de los mayores, se trata de integrarla en otros grupos de menor edad.

3) Que uno de los leitmotiv de la sociedad de consumo es integrarnos en otras categorías sociales, entre las cuales la de la juventud (antítesis de la vejez) se aproxima más al trinomio salud-belleza-vida.

4) Que entre los consumidores mayores habrá rasgos comunes que obedecen también al hecho de compartir aspectos comunes, pero también coexistirán tantas subcategorías - subsegmentos, si seguimos hablando de mercado- como tipologías hay en el resto de consumidores, aunque lógicamente algunas de ellas serán más preeminentes que otras entre los mayores.

Lo que los economistas llaman la elasticidad de la demanda no es más que la confluencia de factores sociales objetivos que inciden en el incremento de la demanda al sumarse distintos colectivos. En lo concerniente al consumidor mayor, se observan, entre otros, los siguientes factores:

A. El creciente número de productos específicos y dirigidos directamente a un consumidor mayor (salud, alimentación, cuidado e higiene, atención, ocio, etc.).

B. El creciente número de productos a los que por primera vez y de manera masiva tienen acceso o se incorporan los consumidores mayores (ocio, comunicación, etc.).

C. La mejora en la imagen social de los mayores y su creciente implicación social a través del consumo (sin negar con esto otros planteamientos más críticos sobre exclusión o «desenganche» social de los mayores).

D. La gran capacidad de la sociedad de consumo de innovar constantemente la oferta de productos, bien sea mejorando los ya existentes o introduciendo otros nuevos.

Como hemos mencionado con anterioridad, el tema del consumo de los mayores debe ser tenido en cuenta no sólo por los Estados que procuran satisfacer las necesidades de este grupo de edad, sino también por las empresas que tienen que estar atentas a los cambios que se producen en el mercado y a la emergencia de nuevos grupos de consumidores cada vez más numerosos con demandas diferenciadas. Si tenemos en cuenta la edad del sustentador principal dentro del hogar, las empresas siempre se han dirigido a un público compuesto por hogares cuyos cabezas de familia fueran menores de 45 años, pero las co- 
sas están cambiando. En opinión de un experto, el grupo que crecerá más rápidamente es el que tiene entre 55 y 64 años, y se calcula que habrá aumentado en más de dos tercios para el año 2010. De ello se pueden sacar tres conclusiones que debieran tener en cuenta las empresas y los especialistas en investigación de mercados (Wallace, 2000: 99-100):

1) La primera es lo absurdo que resulta prever un boom económico según el perfil de consumidor para los próximos diez años a medida que la generación de los nacidos durante el baby boom alcanza su máximo poder adquisitivo.

2) La segunda conclusión es que aquellas empresas que se dedican a ofrecer productos y servicios dirigidos principalmente a los hogares jóvenes se verán en un gran apuro.

3) La tercera es que el envejecimiento de las poblaciones occidentales transformará la composición de la demanda de los consumidores.

En las familias de edad avanzada se observa cómo el poder adquisitivo es más discrecional. Según el ciclo de vida de los hogares, en Gran Bretaña, los cabezas de familia con los hijos ya emancipados poseen la mayor cantidad de coches nuevos, según la empresa de estudios de mercado Mintel. Las personas que acaban de entrar en la sesentena son las que más despilfarran en los hogares cuando se van los hijos; en concreto, son grandes consumidoras de aparatos domésticos y de mobiliario de calidad. Otro hecho es que, al dejar de estar atadas a la casa por los hijos pequeños, están deseosas de utilizar esos coches nuevos para salir e ir a muchos sitios. Como tienen a su disposición más dinero para gastar, los hogares cuyos cabezas de familia tienen entre 55 y 64 años gastan en restaurantes una cuarta parte más que la media. Y, lo que es más importante, estos consumidores dejan de abastecerse de necesidades básicas. Se aficionan más a los servicios que a los productos y prefieren, o requieren, especial atención a la salud, servicios financieros y vivencias relacionadas con el ocio.

Por lo tanto, se observa que a medida que estas generaciones del baby boom alcancen los cincuenta o los sesenta dispondrán de más tiempo y de mucho más dinero. Al igual que la clase del ocio optó por el consumo ostentoso, en la famosa obra de Thorstein Veblen, estas generaciones del ocio gastarán su dinero de muchas formas, afirmando su estatus de personas que se lo han ganado. Esta sed de ocio hará que la industria del turismo sea todo un éxito a medida que la generación del ocio busque nuevas formas de emplear su tiempo. En el Reino Unido, los hogares cuyos cabeza de familia tienen entre 50 y 64 años gastan casi un tercio más que cualquier tipo de hogar cuando sus miembros salen de vacaciones al extranjero. Durante la primera mitad de la década de 1990, el tiempo que las personas mayores de 55 años dedicó a vacaciones aumentó en más de un tercio (Wallance, 2000: 110). 
Un sector bastante beneficiado es la industria naviera. En 1988 se estaban construyendo más de 40 nuevos barcos cruceros; a este auge de los cruceros se ha sumado hasta la Disney. En la última campaña de Disney, en los anuncios, que tradicionalmente iban dirigidos a familias con niños pequeños, ahora aparece un público más viejo; dejan a sus niños grandes en casa para disfrutar ellos mismos, o tienen el nido vacío (Noel, 2001). Otro sector que ha crecido muy rápido en el mercado británico es el de los coches deportivos; las fuentes de la industria sugieren que los miembros de familias sin hijos son los principales consumidores. Los consumidores maduros sostienen el $58 \%$ del ingreso disponible de la nación (Estados Unidos), lo que hace que los minoristas comercien con los más mayores, cambiando la estrategia de mercado, que probablemente hará el mundo de la venta más hospitalario para los seniors.

Muchos otros servicios y productos de lujo, como joyas de la más alta calidad, se deberían beneficiar también de la creciente población de ricos consumidores de edad avanzada. Thorstein Veblen escribía que el consumo ostentoso se reducía a un pequeño grupo de magnates sin escrúpulos del periodo capitalista salvaje del oeste americano; un siglo después, el consumo ostentoso de objetos de lujo se extiende a toda una generación.

Otra industria que atrae a estas generaciones de mayores es la farmacéutica, que se alía con la cosmética. Los farmacosméticos, por lo tanto, suponen un mercado masivo en potencia dirigido por la generación de los nacidos durante el baby boom, conscientes de lo que representa la juventud (Ward, 1998). Se consumen todo tipo de lociones terapéuticas que contienen alfahidroxiácidos derivados de los azúcares y de la leche; los mostradores ya exhiben productos que contienen vitamina $\mathrm{C}$ como reclamo; la ciencia o la cosmética al servicio de mantenerse joven y con el mejor aspecto posible durante más años.

Otra nueva industria emergente es la «nutracéutica» (artículo de comida saludable); el auténtico reto de esta industria se basa en desarrollar una nueva serie de productos alimenticios concebidos científicamente para mejorar la salud. Unilever es una importante empresa pionera en este aspecto. En 1999 lanzó una nueva versión de la margarina Flora, con niveles mayores de esteroles vegetales, que está concebida para reducir el colesterol en la sangre en un 10\% (Unilever, 1988). Muchos consumidores creen que se ata la calidad de su salud directamente a la calidad nutritiva de su dieta, y los mayores es más probable que ajusten sus hábitos de compra basados en los problemas de salud de sus vidas. Casi nueve de cada diez compradores sienten que pueden reducir poco o bastante el riesgo de tener determinadas enfermedades comiendo saludablemente. En el informe «Shopping for Healh 2000", nueve de cada diez consumidores están de acuerdo en que frutas, verduras y cereales contienen sustancias que pueden ayudar a prevenir el cáncer (Kruger, 2001). Dado que las personas mayores están viviendo más y han dado espacio al cuidado pre- 
ventivo, los problemas de salud de los mayores afectan a sus hábitos de compra. La variedad de comidas orientadas a la salud en los supermercados se convierte en algo interesante para alcanzar bienestar y buena nutrición al consumidor maduro, que es el público más indicado para ser atraído con productos bajos en grasa, libres de azúcar, bajos en calorías, etc. (USA Today Magazine, 1998).

Tal como se ha podido comprobar, las empresas farmacéuticas y cosméticas, el sector de los servicios financieros y las industrias dedicadas al ocio son los grandes beneficiarios del envejecimiento de la población.

Existen empresas que tienen que adoptar estrategias diferentes para sobrevivir a la inversión de la pirámide de la población, como la empresa Whitebread, que antes era una empresa cervecera y dueña de sus propios locales y que ahora se ha reinventado a sí misma como empresa de servicios de ocio, con una presencia notable en restaurantes por medio de cadenas como Pizza Hut y Café Rouge. También dirige una cadena de hoteles económicos y una gran cadena de gimnasios. La decisión de Whitebread de pasarse a los centros de salud está pensada para sacar provecho de que la generación del baby boom pretende mantenerse sana y en forma.

Otro fenómeno social de gran importancia para la economía y el consumo son los jubilados residentes en las costas españolas que vienen a pasar los meses más fríos de sus países europeos a España, en busca fundamentalmente del buen clima. En un reciente estudio se destaca la importancia de este fenómeno para las costas murcianas: Un nuevo mercado turístico: jubilados europeos residentes en la Región de Murcia. Se constata cómo, cada vez más, la Región de Murcia se consolida como un destino final o lugar de residencia definitiva para muchos jubilados europeos (Monreal et al., 2001). El número de jubilados extranjeros europeos residentes en la Región de Murcia, según las estadísticas, asciende a 22.000 personas. Este dato debe tomarse como aproximativo ya que estamos ante un hecho que ha crecido bastante en las últimas décadas y seguro que seguirá aumentando en el futuro. Las repercusiones económicas y sociales aún no son conocidas, a diferencia de lo que pasa en otros espacios del litoral español para los cuales se han realizado diferentes investigaciones e informes (Costas Brava, del Sol, Blanca; o de las islas).

El cuerpo y su cuidado, bien sea a través de la alimentación o por el uso de productos de salud y de belleza, es uno de los asuntos que, como se ha comentado, más va a preocupar a nuestros mayores. Los análisis sobre la construcción social del cuerpo y de sus atributos gozan de una cierta tradición en la sociología. A nadie se le escapa el hecho de cómo nuestro cuerpo está sometido a las exigencias sociales, donde la apariencia exterior es objeto de comparación (Turner, 1992: 116-119), donde el aspecto físico es una plusvalía so- 
cial de primer orden en la sociedad de consumo (Sánchez Vera, 1993b). Como señala un experto, «nuestra envoltura corporal, siempre ha estado sometida al rigor de las normas sociales, siquiera éstas ya no impongan su dominio en el interior del cuerpo, allí donde se asientan nuestras pasiones, sino sobre su apariencia exterior convertido en un franco objeto de comparación entre extraños» (Castillo, 1997: 29). En este concreto sentido cabe sostener cómo en las sociedades contemporáneas el cuerpo está sujeto a los preceptos de la llamada racionalidad formal o instrumental, donde existe una «dirección científica del cuerpo y su sujeción a los tornadizos gustos de los estilos de vida» (o.c.: 36). Para Turner, el cuerpo presenta una amplia superficie apropiada para exhibir marcas de posición familiar, rango social, afiliación tribal y religiosa, edad y sexo.

Autores como Bourdieu llamarán la atención sobre el hecho de que la constitución corporal es producto de herencia familiar y social, siendo el cuerpo un exponente de la posición en nuestra sociedad. Este autor va más lejos al relacionar los patrones físicos del cuerpo ideal con los valores de los grupos dominantes, poniendo el ejemplo de la relación entre los movimientos y ademanes corporales y las clases sociales (Bourdieu, 1986), según lo cual el cuerpo humano será un factor significativo en la lucha de clases (o.c.: 186-188). Para otros autores, estas tradicionales divisiones por clases sociales, o las actuales por grupos de estatus, no son suficientes para explicar la gran variedad de estilos de vida (Featherstone, 1991, y Klapp, 1969). A propósito de esto, el profesor Castillo subraya la importancia que hoy tienen los estilos de vida en la sociedad, y cómo el cuerpo es exponente más del estilo de vida que de la posición social (o.c.: 41-42).

\section{CONCLUSIONES}

El asunto de los mayores y el consumo es un tema de creciente interés científico, en la medida en que cada vez son más en número y en influencia social (y económica) los consumidores mayores, y a los que sin duda, y de manera inexorable, habrá que ir prestando una mayor atención -y no sólo como usuarios de servicios sociales diversos-, en tanto que constituyen cada vez más un segmento diferenciado de consumidores que mueve un importante volumen de dinero en la economía española.

Es cierto que la edad $-\mathrm{y}$ las experiencias generacionalmente vividas y acumuladasmarca al sujeto en sus actitudes ante la vida, pero hay muy diferentes tipos de mayores con actitudes vitales igualmente distintas. $Y$ en el asunto del consumo que aquí nos ocupa, dichas actitudes van a mediatizar la propia estructura de consumo que cada sujeto va a tener. Estas actitudes vitalistas para consigo mismos, y en donde hay un proyecto de vida propio (por fortuna, cada vez más presente entre los mayores) en el que hacen y disfrutan 
de bienes y consumos a los que antes nunca pudieron tener acceso (bien por falta de dinero o por circunstancias familiares), no tienen por qué estar enfrentadas — de hecho, así suele ocurrir - con el altruismo y generosidad para con los suyos.

Ante todo, queremos hacer ver que, siendo verdad que hay rasgos comunes en las personas mayores ante el consumo, si algo sabemos los sociólogos es que hay sujetos concretos con sus propios valores y circunstancias objetivas. Junto con la edad (los hay de diferentes edades) y la situación económico-patrimonial, el nivel de estudios, el estado de salud, el género, la situación familiar y la biografía laboral de cada sujeto constituyen otros tantos elementos a considerar a la hora de conocer al consumidor mayor. Así, este segmento de consumidores, teniendo algunos rasgos comunes entre sí, sin embargo, presenta diferencias y características entre subgrupos (o subsegmentos).

El mayor no es uno de los grandes objetivos del consumo, partiendo de la idea, no siempre acertada, de que dispone de escaso poder adquisitivo pero va ganando poco a poco espacio y atención. En efecto, son mayoritarios los mayores con recursos escasos, que tienen que hacer un minucioso control del gasto para llegar a fin de mes, pero también es verdad que existen mayores que en términos reales poseen un poder adquisitivo igual o incluso más alto que el que disponen otros grupos de jóvenes, los cuales perciben unos ingresos sensiblemente inferiores a los de los mayores. El hecho de tener una casa pagada y unos hábitos de consumo «controlados» es un capital adicional importante. Tampoco hay que desmerecer el hecho de que también algunos mayores cobran pensiones relativamente importantes, máxime teniendo en cuenta el alto porcentaje que tiene la casa pagada. No es extraño tampoco el que la propia pensión (y la del cónyuge) tenga complementos provenientes de ahorro o de otras rentas.

En general, y a pesar de la segmentación de mercados, los mayores no han sido todavía abordados por los sociólogos y los economistas especializados en la investigación de mercados con la decisión que un sector tan importante de la población merecería. Los mayores constituirán a buen seguro un nicho de producción importante en los próximos años, donde existirán cada vez más productos especializados para ellos (al igual que los hay para niños, jóvenes, etc.), y donde se les preste más atención tanto desde el campo del consumo como de la publicidad. 


\section{BIBLIOGRAFÍA}

AGUIAR, F.; PÉREZ YRUELA, M., y SERRANO, R. (1995): Indicadores sociales de calidad de vida y Tercera Edad, Madrid, Instituto de Estudios Sociales Avanzados (IESA), Documento de Trabajo 95-05.

ALFAGEME, A. (1999): «Envejecimiento y desigualdad social», Revista Internacional de Sociología, tercera época, n. ${ }^{\circ} 24$, pp. $121-135$.

ALONSO, L. E., y CONDE, F. (1994): La historia de la sociedad de consumo en España, Madrid, Ed. Debate.

ALTHUSSER, L. (1969). «El objeto del capital», en L. Althusser y E. Balibar, Para leer El Capital, México, Siglo XXI.

BARAN, P. (1957): «A Marxist View of Consumer Sovereight», Prólogo a The Political Economy of Growth, Montheley Review Press. Reimpresión Bobs-Merrill.

BAUDRILLARD, J. (1976): La génesis ideológica de las necesidades, Barcelona, Anagrama.

BAZO, M. ${ }^{a}$ T. (1996): “Aportaciones de las personas mayores a la sociedad: análisis sociológico», REIS, $\mathrm{n} .^{\circ} 73$, pp. 209-222.

BLUMER, H. (1982): El interaccionismo simbólico, Barcelona, Hora.

BOURDIEU, P. (1986): «Notas provisionales sobre la percepción social del cuerpo», en VV.AA., Materiales de Sociología Crítica, Madrid, Las Ediciones de la Piqueta.

- (1988): La distinción (criterios y bases sociales del gusto), Madrid, Tecnos.

CASTILLO, J. (1997): «El cuerpo recreado: La construcción social de los atributos corporales», Revista de Pensamiento Social, $\mathrm{n} .^{\circ} 2$.

CIS-IMSERSO (1998): La soledad de las personas mayores, Estudio n. ${ }^{\circ} 2279$.

CONDE, F. (1994): «Notas sobre la génesis de la sociedad de consumo en España», Política y Sociedad, n. ${ }^{\circ} 16$, pp. 135-148.

CUMMING, E., y HENRY, W. H. (1961): Growing Old. The process of didengagement, Nueva York, Basic Book.

DOMÉNECH, A. (1985): «La crisis desde el punto de vista de las necesidades humanas», Mientras Tanto, $\mathrm{n} .{ }^{\circ} 22$, pp. 49-59.

DOYAL, L., y GOUGH, I. (1991): A Theory of Human Nedd, Londres, MacMillan.

DUFF, M. (1998): «Seniors», Discount Store News, vol. 37, Issue 20, p. 102.

FEATHERSTONE, M. (1991): Consumer culture and postmodernism, Londres, Sage Publications.

GAVIRIA, M. (1996): La séptima potencia, Barcelona, Ediciones B.

GIL, E. (1992): «La emancipación de los ancianos», en L. Moreno y M. Pérez Yruela (comps.), Política Social y Estado de Bienestar, Madrid, Ministerio de Asuntos Sociales.

GRANDE, I. (1993): Marketing estratégico para la Tercera Edad, Madrid, ESIC.

GURBRIUM, J. F. (1973): The myth of the golden years, Illinois, Thomas Springfield.

HALBWACHS, M. (1970): La clase ouvriére et les niveaux de vie, París, Gordon and Breach. 
HAVIGHURT, R. J., y ALBRECHT, R. (1953): Older people, Nueva York, Longman.

INSTITUTO NACIONAL DE CONSUMO (2000): La tercera edad y el consumo, Madrid.

INSTITUTO NACIONAL DE ESTADÍSTICA (2001): Encuesta sobre Discapacidades, Deficiencias y Estado de Salud.

KLAPP, O. E. (1969): Collective search for identity, Nueva York, Holt, Rinchart and Winston, Inc.

KRUGER, R. M. (2001): «Senior Health Food Watch», Retail Merchandiser, vol. 41, Issue 3, p. 40.

LACLAU, E., y MOUFFE, C. (1987): «Post Marxism whitout apologies», New Left Review, n. ${ }^{\circ} 166$.

LEVANTHAL, R. C. (1997): Journal of Consumer Marketing, vol. 14, Issue 4/5, p. 276.

MANNHEIM, K. (1993): «El problema de las generaciones», REIS, n. ${ }^{\circ}$ 62, pp. 193-242.

MASLOW, A. H. (1954): Motivación y personalidad, Barcelona, Sagitario.

MARX, K. (1968): El Capital, México, FCE.

MINISTERIO DE SANIDAD Y CONSUMO (1997): Encuesta Nacional de Salud.

MONREAL MARTÍNEZ, J.; FRUTOS, L.; GUARDIOLA, P.; MELLADO, M.; ORTíN, J.; PÉREZ, C.; SÁNCHEZ VERA, P., y VERA REBOLLO, J. F. (2001): Un nuevo mercado turístico: jubilados europeos residentes en la Región de Murcia, Murcia, J. Monreal Martínez (ed.), Universidad de Murcia.

MORAGAS, R. (1991): Gerontología Social (envejecimiento y calidad de vida), Barcelona, Herder.

NAVARRO, M. (1987): «Producción y Consumo», en J. A. Garmendia, M. Navarro y F. Parra Luna, Sociología Industrial y de la empresa, Madrid, Ed. Aguilar.

NOEL, P. (2001): «Selling to Seniors», Christian Science Monitor, vol. 93, Issue 78, p. 11.

ORTÍ, A. (1976): «Estratificación social y estructura de poder», en Homenaje a F. Murrillo, Madrid, CIS.

PÉREZ, L. (1998): Las necesidades de las personas mayores. Vejez, economía y sociedad, Madrid, Ministerio de Trabajo y Asuntos Sociales.

PÉREZ-AGOTE, A. (1978): «Hacia un estatuto teórico del consumo: Jean Baudrillad o la abstracción lógica de la forma», en J. Jiménez Blanco y C. Moya, Teoría Sociológica Contemporánea, Madrid, Tecnos.

RUIZ DE MAYA, S. (1999): «Los grupos y el análisis de la familia», en J. A. Rivas et al., Comportamiento del consumidor, Madrid, ESIC, cap. 7.

SÁNCHEZ, P. (1993a): «Homogeneidad y diferenciación en la tercera edad. Bases para una sociología de la ancianidad», en P. Sánchez Vera (ed.), Sociedad y Población Anciana, Murcia, Ed. Universidad de Murcia, pp. 31-52.

— (1993b): «Bases y fundamentos para una aproximación sociológica a la vejez», Papers, n. ${ }^{\circ} 40$.

- (2000): «Sociología de la vejez versus economía de la vejez», Papers, n. ${ }^{\circ} 61$, pp. 39-88.

- (2001): «Construcción social de los hábitos de consumo en las personas mayores», Cuadernos de Realidades Sociales, n. ${ }^{\text {os }} 57 / 58$, pp. 95-123.

SÁNCHEZ, P., y BÓDALO, E. (2000): «Presencia y ausencia de los mayores en la publicidad televisiva», Revista del Ministerio de Trabajo y Asuntos Sociales, n. ${ }^{\circ}$ 25, pp. 19-47. 
SEN, A. (1984): «Poor, relatively speaking», en Sen, Resources, Values and Development, Oxford, Balckwell.

SMITH, G. (1980): Social Need: Social Practice and Research, Londres, Routledge.

TURNER, B. S. (1992): Max Weber. From history to modernity, Londres, Routledge.

UNILEVER (1988): Possitive assessment in the Netherland Brings New Flora Pro-activ Closer to the Market. USA TODAY MAGAZINE (1998): “Catering to older consumer», vol. 127, Issue 2643, p. 13.

WALLACE, P. (2000): El seísmo demográfico, Madrid, Siglo Veintiuno de España Editores.

WARD, V. (1988): «Acquiring a Taste for Looking Good», Financial Times Pharmaceutical Survey.

In this article we have made an analysis of the various ideas of needs experienced in the population segment that covers those over the age of 65 , and we can confirm that although this population group has the same needs as all other age groups, it is also possible that they may need another series of goods and services that differentiate them from other population segments.

Our aim is to highlight the way in which the time factor affects the consumer habits of the elderly, since they have lived through different times or situations for reasons of their age, the end result being the sum of the times lived through and the personal situation of each individual at any given moment in time.

Lastly, an attempt is made to see how the effects of the generation they belong to, as well as the various family life cycles, have an influence on their situation regarding consumer habits; emphasis is placed on the fact that this segment contains an ever-increasing number of elderly consumers. 